\title{
Application of the Image Subtraction Method to the OGLE-I Observations
}

\author{
Grzegorz Kopacki, Zbigniew Kolaczkowski, Andrzej Pigulski \\ Wroctaw University Observatory, Kopernika 11, 51-622 Wroctaw, \\ Poland
}

\begin{abstract}
The analysis of the OGLE-I data using the standard profilefitting software yielded a large set of new variable stars. However, due to the severe crowding of the images many variables may have been missed. Therefore, we decided to reanalyze these data by means of the image subtraction method. Our main goal was the detection of new variables. In addition, we intend to extend the time-base of the observations for the variables found in the course of the OGLE-II project (Udalski et al. 1997). We present the preliminary results of our study.
\end{abstract}

\section{Introduction}

The Optical Gravitational Lensing Experiment (OGLE, Udalski et al., 1992), which just began the third stage of its operation, brought a very large amount of observational data.

The search for variable stars in the OGLE-I data was performed by means of the standard point-spread-function (PSF) fitting software. It yielded a large set of new variables, including many pulsators of the RR Lyrae, SX Phoenicis, and $\delta$ Scuti type (Udalski et al., 1997). However, due to the severe crowding many variables may have been missed.

\section{The method}

As was shown by many authors, the Image Subtraction Method (ISM) developed by Alard \& Lupton (1998) works better than the PSF fitting in crowded fields and is extremely effective in finding variable stars, even in the cores of globular clusters (see, e.g., Kopacki, 2001).

Despite the well-known defects of the CCD chip used in the OGLE-I observations, we decided to reanalyze these data by means of the ISM method. Our main goals were the detection of new variables and the improvement of the photometry of the already known ones. Additionally, we intend to extend the time-base for variables which were or will be found within the OGLE-II project (Udalski et al., 1997). The software we use is a combination of the ISM code provided by Dr. C. Alard, the Difference Image Analysis (DIA) code developed recently by Dr. P.R. Woźniak (Woźniak, 2000), and our own programs. 


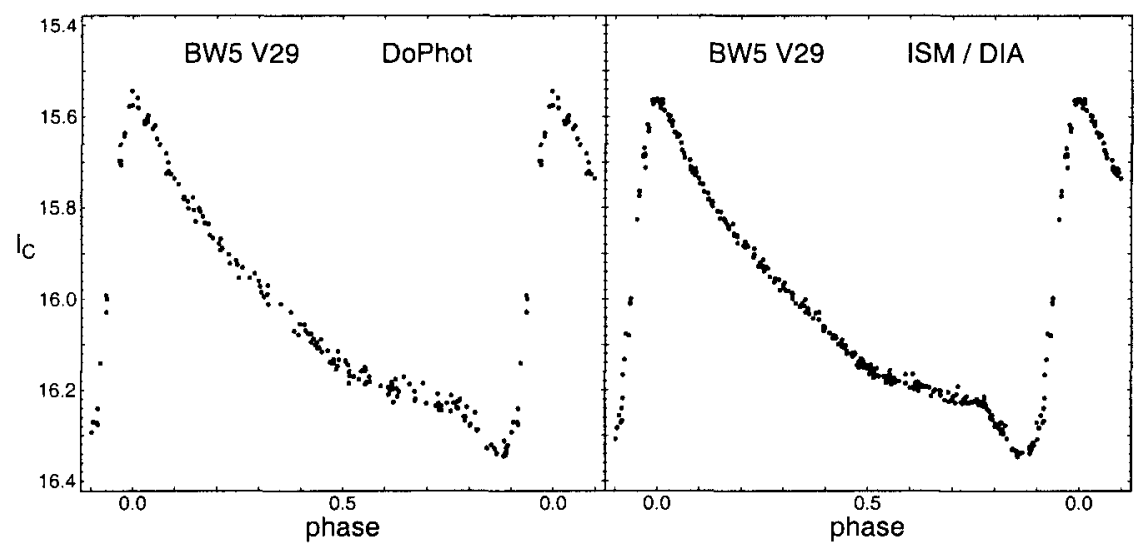

Figure 1. The $I_{\mathrm{C}}$-filter light curves of the RR Lyrae star BW5 V29 = V1346 Sgr obtained with the profile-fitting package DoPhot (left panel) and with the combined ISM/DIA method (right panel). Note significant improvement in the photometric quality.

\section{Some results}

To test our combined method we reduced the full set of observations of one of about 30 OGLE-I fields, namely BW5 field in the Baade's Window. It covers rougly an area of $4^{\prime} \times 4^{\prime}$.

We were able to detect all the already known pulsating stars in the field and moreover we found many new variables. As an example, we show in Fig. 1 the light curve of the RRab variable BW5 V29 = V 1346 Sgr. It can be seen that even for a well-isolated star like this one, a substantial improvement in photometric quality is obtained when compared with published DoPhot photometry. The analysis of the whole OGLE-I set of images is underway.

Acknowledgments. This work was supported by KBN grant 2P03D 006 19.

\section{References}

Alard, C. \& Lupton, R.H. 1998, ApJ, 503, 325

Kopacki, G. 2001, A\&A, 369, 862

Udalski, A., Kubiak, M., \& Szymański, M. 1997, Acta Astron., 47, 319

Udalski, A., Szymański, M., Kałużny, J., Kubiak, M., \& Mateo M. 1992, Acta Astron., 42, 253

Woźniak, P.R. 2000, Acta Astron., 50, 421 